
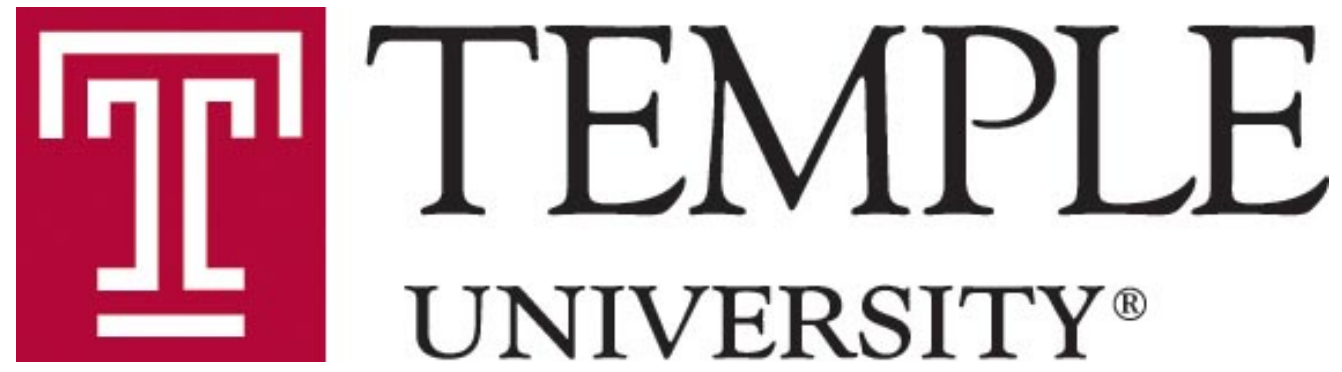

\title{
Structural Models, Information and Inherited Restrictions
}

\author{
by \\ Andrew J. Buck \\ George M. Lady \\ Department of Economics \\ DETU Working Paper 11-03 \\ June 2011
}

1301 Cecil B. Moore Avenue, Philadelphia, PA 19122 http://www.cla.temple.edu/economics/ 


\section{Structural Models, Information and Inherited Restrictions}

\section{By}

\section{George M. Lady and Andrew J. Buck*}

JEL Classification: C15, C18, C51, C52

Key Words: Qualitative analysis, structural form, reduced form, identification, entropy, model falsification

Word Count: 11,696

Page Count: 40

*Both authors are Professor of Economics, Temple University, Philadelphia, PA 19122

Corresponding Author: Andrew Buck, buck@temple.edu, 215-646-1332. 


\title{
Structural Models, Information and Inherited Restrictions \\ By George M. Lady and Andrew J. Buck
}

\begin{abstract}
The derived structural estimates of the system $\beta Y=\gamma Z \| \delta U$ impose identifying restrictions on the reduced form estimates ex post. Some or all of the derived structural estimates are presented as evidence of the model's efficacy. In fact, the reduced form inherits a great deal of information from the structure's restrictions and hypothesized sign patterns, limiting the allowable signs for the reduced form. A method for measuring a structural model's statistical information content is proposed. Further, the paper develops a method for enumerating the allowable reduced form outcomes which can be used to falsify the proposed model independently of significant coefficients found for the structural relations.
\end{abstract}




\section{Structural Models, Information and Inherited Restrictions \\ By George M. Lady and Andrew J. Buck}

I. Introduction. In his Foundations (1947) Samuelson proposed that economic theory should be understood to organize aspects of how the economy works by mathematical models expressed by systems of equations:

$$
\mathrm{f}^{\mathrm{i}}(\mathrm{Y}, \mathrm{Z})=0, i=1,2, \ldots, n,
$$

where $\mathrm{Y}$ is an n-vector of endogenous variables and $\mathrm{Z}$ is an m-vector of exogenous variables. The system is studied by, and the potential for its acceptance or rejection resides in, the method of comparative statics. Such analyses assess the effects of changes in the entries of $Z$ on the entries of $\mathrm{Y}$ with respect to a referent solution as specified by a linear system of differentials:

$$
\sum_{j=1}^{n} \frac{\partial f^{i}}{\partial y_{j}} d y_{j}+\sum_{k=1}^{m} \frac{\partial f^{i}}{\partial z_{k}} d z_{k}=0, i=1,2, \ldots, n .
$$

Refutation, or not, of the theory is then taken up by the econometrician. In econometrics, the relationships in (1) and (2) are often assumed to be (at least locally) linear; and, with the inclusion of an unobserved disturbance (2) can be represented by the linear system,

$$
\beta Y=\gamma Z,+\delta U
$$

where $\beta, \gamma$ and $\delta$ are appropriately dimensioned matrices. (3) is usually called the structural form of the model. In macroeconomics this rather general form could just as easily represent a model such as Klein's (1950) Model I or a structural vector autoregression (SVAR) model such as Sims (1986). ${ }^{1}$ These models constitute rather different, competing views about what the economist is presumed to know and how the macroeconomy works. As a matter of implementation, the distinction between the two approaches to modeling the economy is a question of identifying

\footnotetext{
${ }^{1}$ Klein's Model I is the quintessential Keynesian model. The SVAR is inclusive of monetarist, real business cycle and neo-Keynesian models. A representative example of these distinctions would be Blanchard and Quah (1989).
} 
restrictions imposed on $\beta, \gamma$ and $\delta$ for purposes of identification. In fact, using the basic model of (3) the Keynesians, the monetarists, the real business cycle theorists and the neo-Keynesians all can claim to be consistent with the data; that is, each can point to statistically significant results for some part of $\beta, \gamma$ and $\delta$ based on the manipulation of the empirical reduced form of (3) given by

$$
\mathrm{Y}=\pi \mathrm{Z}+\psi \mathrm{U}
$$

where $\pi=\beta^{-1} \gamma$. The student of economics is left with a conundrum: Who is right? Are any of them right? What is the state of economic science if none of the competing models can be refuted on the basis of test statistics?

It is generally agreed that a field organizes its subject matter "scientifically" if it presents substantive propositions that can, in principle, be refuted. The theory of a "scientific" discipline must call for some sort of limits on what the data outcomes can be; so that, if a disallowed outcome is observed, then the theory is falsified (Popper (1934)). The more the theory imposes limits on the possible outcomes, the more (as we propose below) information it provides. And, as more information is provided, the potential for falsification is greater, and the success of the theory, i.e., if observations consistently do not find the disallowed outcomes, is to that degree more substantial.

This paper confronts the issue of the falsification of economic models in much the same way that Samuelson (1947) did; namely, by recognizing that economic theory's propositions about the system (3) are seldom, if ever, quantitatively complete. Specifically, with occasional exceptions, the theory usually only proposes the sign patterns of $\beta$ and $\gamma$. Accordingly, the issue of testing the theory requires that such sign pattern information lead to limitations on the outcome of estimating $\pi$. Samuelson didn't feel that it was very likely that the restrictions on $\beta$ and $\gamma$, if only expressed as sign patterns, could be carried through to limiting the empirical outcomes for $\pi$. Still, a literature found the very restrictive conditions for a mapping from the sign pattern of $\beta$ and $\gamma$ to $\pi$ (a qualitative analysis). The conditions are so restrictive that such analyses are of extremely limited use in practice. 
We propose to revisit the issue of qualitative analysis for the purpose of testing a specification of $\beta$ and $\gamma$ in terms of the limits imposed on the permissible empirical outcomes for $\pi$. We show that qualitative information about the system (3) always (rather than virtually never) provides substantial information concerning the limits on the outcomes for estimating $\pi$. Further, in principle, the possible outcomes for $\pi$ based upon a qualitative specification of (3) can be expressed as a frequency distribution of possibilities; and, as such, the "information" contained in the model's structural specification can be based upon the Shannon entropy of the distribution. Compared to current practice, this enables two remarkable capabilities: Any qualitative specification of (3) can be brought to the data and potentially falsified by the reduced form; and, the entropy of alternative specifications can be compared on the basis of the limitations imposed by the specification on the possible outcomes for $\pi$.

The paper is organized as follows: In section two the notion of falsification in modeling is discussed. The meaning of a qualitative inverse and qualitative falsification are introduced in section three. Section four uses Shannon's Information Measure to calculate the entropy of a model and to calculate the information contained therein, vis-à-vis what can be learned from the data. The Monte Carlo technique that we use to model the possible outcomes for the reduced form and to possibly falsify the structural model is contained in section five. In section six we present three examples of our method of assessing a model's consistency with the data and its information content. The examples are Klein's Model I, Sim's 1986 structural vector autoregression and the Department of Energy's Oil Market Simulation. Klein's model was chosen because it admits possible falsification of the model through the identifying restrictions imposed on the model's endogenous and exogenous coefficient matrices. Sims introduced his model as a bridge between the considerable economic knowledge presumed in a model like Klein's and the absence of economic knowledge presumed in the early vector autoregressions. Sims' model is of particular interest because it admits the possibility of falsification through the identifying restrictions placed on the error covariance matrix while essentially ignoring hypothesized signs for the structural exogenous coefficients. The last example, the Oil Market Simulation (OMS), is of interest for several reasons. First, it is amenable to the qualitative analysis that our paper supersedes. Second, although the OMS is not falsified by the historical 
qualitative approach, our approach does falsify it. Lastly, as derived, the OMS can be subjected to falsification analysis as either a structural multi-market model or as a structural vector autoregression.

II. Falsification in Practice. Econometric practice manipulates the outcome of estimating an unrestricted version of $(4)^{2}$ to recover the implied arrays $\{\beta, \gamma\} .^{3}$ In doing this, the practitioner is confronted with the problem of identification. If (3) is under-identified, then the complete recovery of the arrays in (3) can be problematical. If (3) is over-identified, then there is more than one method available to use in recovering (3) and the results for each of them may not be in agreement. ${ }^{4}$ For identified systems, the restrictions in the hypothesized $\{\beta, \gamma\}$ are uniquely present in the derived estimates of these arrays. All of this fails to be decisive as now practiced in the sense that it is unusual for a model to be "falsified" by the outcome of recovering $\{\beta, \gamma\}$ from the estimation of (4). Although "problems" may be encountered, they are as likely to be viewed as econometric rather than related to the validity of the conceptual model. ${ }^{5}$

An issue to resolve is whether the theoretical propositions about $\{\beta, \gamma\}$ could be potentially falsified by the estimated reduced form (4). As Samuelson noted, economic theory does not typically propose magnitudes for the entries of the hypothesized $\{\beta, \gamma\}$. Usually, only the signs $(+,-, 0)$ are proposed and we are left with the need to implement a qualitative analysis; namely, finding restrictions on the outcome of estimating (4), based upon the hypothesis in (3), that can be applied directly. Technically, this amounts to deriving limitations on the outcome of estimating $\pi$, i.e., its sign pattern, based upon a knowledge of the sign patterns in $\{\beta, \gamma\}$. Since he thought that a successful qualitative analysis would rarely be possible, Samuelson proposed ways to work around the difficulties using other points of view, e.g. the correspondence principle. However, none of Samuelson's approaches have evolved into common econometric practice. ${ }^{6}$

\footnotetext{
${ }^{2}$ To our knowledge the only instance of carrying the implied sign restrictions of $\beta$ and $\gamma$ forward to the estimation of the reduced form is in Berndt's (1990) discussion of Goldberger (1964). However, in spite of Berndt's discussion, it doesn't appear to us that this was actually done by Goldberger.

${ }^{3}$ Instrumental variables, two stage least squares and three stage least squares would be examples.

${ }^{4}$ As an example consider Greene's (2008) discussion of Klein's Model I. This discussion is common to every edition of this popular textbook.

${ }^{\mathbf{5}}$ Leamer (1983) spawned the beginnings of these considerations with his parable about the farmer at the American Ecological Association. The issue is also taken up in Hendry (1980) and Gilbert(1986).

${ }^{6}$ Samuelson further proposed that the LeChatelier Principle would be exhibited in the solutions to optimization problems (op. cit.) and, sometimes, in multimarket equilibria, Samuelson (1960). There is a literature on this, more
} 
Instead, the evaluation of the theory is usually related to model fit, and the often problematical recovery of $\{\beta, \gamma\}$ seldom plays a decisive role. Whatever is done is virtually never qualitative.

The literature on the conditions under which a qualitative analysis can be successfully conducted (typically) considers a special case of (3) and (4) in which $n=m$ and $\gamma$ is the identity matrix. Lancaster (1962) provided sufficient conditions for the form of $\beta$ 's sign pattern that allowed at least some of the signs in $\beta^{-1}$ to be determined. Basset, Maybee, and Quirk (1968) provided necessary and sufficient conditions for a successful qualitative analysis for the sign pattern of $\beta$ put into a standard form. Lady (1983) provided similar necessary and sufficient conditions for a successful qualitative analysis for $\beta$ 's sign pattern put into a slightly weaker standard form, plus algorithmic principles for constructing such systems. ${ }^{7}$ Starting with Lancaster (op.cit), there was attention in the literature to the problems of conducting a successful qualitative analysis. A good deal of this is cited in Hale, et al (1999). The conditions on the sign patterns of $\{\beta, \gamma\}$ that allow at least some of the signs of the entries of $\pi$ to be necessarily determined are well in-hand. Significantly, the analysis is limited to finding specific signs in $\pi$.

None of this literature on the mathematics of qualitative falsification dispelled Samuelson's original observation that a successful or widespread qualitative analysis was unlikely. Indeed, the literature on attempts to conduct a qualitative analysis is sparse (e.g., Ritschard (1983), Maybee and Weiner (1988), Hale and Lady (1995), Lady (2000), Buck and Lady (2005)). As practiced, the qualitative analysis of an actual model provided a useful inspection of the inference structure of the model; however, it was in general an open ended process of considering special cases utilizing other information in addition to the sign patterns of $\{\beta, \gamma\}$. There is no tradition of any kind that we know of for using a qualitative analysis, however modified, to (potentially) falsify economic models.

focused on detecting the presence of the Principle, rather than testing the Principle as an hypothesis. See Lady and Quirk (2007 and 2010). Otherwise, we know of no extensive literature on the rest of it, with some exceptions noted in the next section.

${ }^{7}$ Actually, Lancaster (1962) and Lady (1983) were studying a slightly different qualitative problem: the conditions under which the sign pattern of $Y$ could be determined based upon the sign pattern of $\beta^{-1}$ and $Z$, i.e., the conditions under which at least one entire column of $\beta^{-1}$ could be determined based upon sgn $\beta$. Lady and Maybee (1983) showed that sometimes, although some entries of $\beta^{-1}$ could be signed, nevertheless no entire column could be signed. 
The paucity of empirical papers applying the concept of qualitative analysis is easily understood. The entire literature on qualitative analysis, starting with Samuelson to the current day, is very restrictive and requires that individual entries of $\pi$ be signable, independent of the rest. This fails to take into account that an hypothesis provided by the sign patterns of $\{\beta, \gamma\}$, or even in part by simply knowing that some entries are nonzero and some of them not, can provide considerable information about (i.e., impose limitations upon) $\pi$ 's possible sign patterns without requiring that any particular entry is limited to a particular sign. We show below that any hypothesis regarding the signs of $\{\beta, \gamma\}$ imposes limits on $\pi$ 's sign pattern and can be potentially falsified. Further, the hypothesis can have its information content measured; and. given this, be compared to alternative hypotheses in terms of its "scientific" content.

III. Qualitative Falsification. In the development of concepts and examples below our scope is limited to computationally non-singular instances of $\beta$. This is not overly restrictive from the perspective of either the mathematical content of the approach or its practical significance. To now, a successful qualitative analysis usually required that $\beta$ be sign non-singular ${ }^{8}$, the conditions for which are very much more stringent than the quantitative restrictions on invertibility. Accordingly, a significant departure of our approach compared to the extant qualitative analysis literature is that we consider matrices which don't necessarily meet the criteria for sign non-singularity and could conceivably be singular. In general, almost all actual applied models could be singular and therefore cannot be studied qualitatively, but of course are not singular in practice. ${ }^{9}$

In addition, to facilitate the analysis, we will assume that $\beta$ is irreducible, i.e., that no entries of $\beta^{-1}$ must be zero. We additionally assume that no entries of $\beta^{-1}$ are otherwise computationally equal to zero. Finally, the arrays $(\beta, \gamma)$ are specified as follows:

(a) which entries are zero and which not;

(b) the signs of (at least some of) the nonzero entries; and,

\footnotetext{
${ }^{8}$ I.e., the matrix is demonstrably nonsingular based only upon its sign pattern.

${ }^{9} \beta$ singular in either the numerical or sign sense would mean that the econometric model was not correctly specified from the start.
} 
(c) distributional rules to which the values of the nonzero entries must conform. ${ }^{10}$

Given this, let $\operatorname{CQ}(\beta, \gamma)$ be the set of all quantitative realizations of $\{\beta, \gamma\}$ consistent with $\beta$ nonsingular and the assumptions (a), (b), (c) above. Let RF(sgn $\pi$ ) be the set of sign patterns for the corresponding reduced forms, where $\pi=\beta^{-1} \gamma$. The issues to resolve are: $\operatorname{Given} \boldsymbol{C Q}(\boldsymbol{\beta}, \boldsymbol{\gamma})$, what are the members of RF(sgn $\pi)$ and what are the frequencies of their occurrence?

As an example, let $\mathrm{n}=\mathrm{m}=2, \gamma=\mathrm{I}$, and the hypothesis is,

$$
\operatorname{sgn} \beta=\left[\begin{array}{ll}
- & + \\
+ & -
\end{array}\right] .11
$$

Further, let the absolute values of each entry of $\beta$ be randomly chosen from the uniform distribution,

$$
0<\operatorname{abs}\left(\beta_{\mathrm{ij}}\right)<10
$$

For this simple example it is easy to see that when $\beta$ is non-singular, as it almost inevitably is, its determinant will be positive or negative, each half of the time. Accordingly, each of the entries of $\beta^{-1}$ will be all positive or all negative, each half of the time. For a traditional qualitative analysis, that generally would be the end of the story. The given sgn $\beta$ is not sign nonsingular and none of the entries in $\beta^{-1}$ can be conclusively signed. ${ }^{12}$ Nevertheless, there is quite a bit of information provided by $\operatorname{sgn} \beta$ about the characteristics of $\operatorname{RF}(\operatorname{sgn} \pi)$. Specifically, $\beta$ 's adjoint is

\footnotetext{
10 The distributional rule is in the nature of a Bayesian prior. The only stipulation is that the prior not admit values for the matrix' entries that violate the proposed sign pattern. To facilitate the examples presented here a uniform distribution is assumed. It is in no way intended to limit the analysis to the assumption of uniform distributions. In any event, subject to scaling, our approach actually enables the consideration of any quantitative realization of a given sign pattern. Hence, the results found are independent of magnitudes for the sign patterns analyzed.

${ }^{11}$ Strictly, sgn $\mathrm{a}=1,-1$, or 0 as $\mathrm{a}>0$, $\mathrm{a}<0$, or $\mathrm{a}=0$. We will use the symbols,,+- 0 instead to facilitate the presentation.

${ }^{12}$ Of course for this simple $2 \times 2$ case the fact that $\beta$ 's adjoint is known would presumably be taken into account; and, similarly for larger systems, e.g., Buck and Lady (2005). Still, in general, if no entry of $\pi$ can be signed, a qualitative analysis is usually considered to have failed and is abandoned unless other quantitative information is added.
} 
entirely signed (although our ideas do not depend on this) and has all negative entries. As a result, $\operatorname{sgn} \beta^{-1}$ can only be all positive, or all negative, each half the time. A 2 x 2 matrix, barring zeros and with no sign restrictions, can have any of sixteen sign patterns. For our example, the hypothesis $\operatorname{sgn} \beta$ and $\gamma=\mathrm{I}$, limits the members of $\mathrm{RF}(\operatorname{sgn} \pi)$ to just two of the sixteen possibilities, each appearing half the time. That is, the hypothesis $\operatorname{sgn} \beta$ precludes any outcomes for $\beta^{-1}$ other than the ones in which the elements of $\beta^{-1}$ are either all negative or all positive.

The method is not limited only to expressions of the sign patterns of $\{\beta, \gamma\}$. Any additional information about these entries provided by the model embedded in (3), or conjectured by a practitioner, can be used to determine what limits are placed on the members of RF(sgn $\pi)$ and the corresponding frequencies of their occurrence. Further, less rather than more information may be processed. As before let $\mathrm{n}=2, \gamma=\mathrm{I}$, and

$$
0<\operatorname{abs}\left(\beta_{\mathrm{ij}}\right)<10
$$

But now, let,

$$
\operatorname{sgn} \beta=\left[\begin{array}{ll}
- & ? \\
+ & -
\end{array}\right]
$$

In this case, the signs of $\beta$ are as before with the exception that, besides being nonzero, the sign of $\beta_{12}$ is unknown. Assume for the example that the sign of $\beta_{12}$ can be positive or negative, each $50 \%$ of the time. When $\beta_{12}$ is positive, then the possibilities for the sign pattern of $\beta^{-1}$ are as before, all positive or all negative, each half of the time. When $\beta_{12}$ is negative, then $\beta$ is sign nonsingular and only one sign pattern for $\beta^{-1}$ is possible. That is,

$$
\text { if } \beta_{12}<0 \text {, then } \operatorname{sgn} \beta^{-1}=\left[\begin{array}{ll}
- & + \\
- & -
\end{array}\right] \text {. }
$$

Now, $\mathrm{RF}(\operatorname{sgn} \pi)$ contains three sign patterns: all positive and all negative, each $25 \%$ of the time; and, the above sign pattern $50 \%$ of the time. The hypothesis now forbids thirteen of the sixteen 
possible sign patterns for $\pi$. If any of these thirteen present themselves at the time of estimation of $\pi$, then the hypothesis is falsified.

We will present examples involving larger arrays below. The simple examples in this section are, nevertheless, sufficient for posing the definition of qualitative falsifiability. Specifically:

Qualitative Falsifiability: The hypothesis given by $\operatorname{sgn}\{\beta, \gamma\}$ is qualitatively falsifiable if and only if there exists a sign pattern $\operatorname{sgn} \pi^{*} \operatorname{such}$ that $\operatorname{sgn} \pi^{*} \notin \mathrm{RF}(\operatorname{sgn} \pi)$.

Simply said, for a given $\operatorname{sgn}\{\beta, \gamma\}$, if it can be shown that there exists a reduced form sign pattern that is impossible, then the hypothesis can be falsified; that is, rejected if the impossible reduced form sign pattern is found from the data. ${ }^{13}$ This definition picks up all cases for which some number of entries in $\pi$ can actually be signed as provided for in the literature on qualitative analysis. But now, the scope of cases that can be approached is substantially enlarged beyond the few instances of economic models satisfying the conditions for sign nonsingularity.

Indeed, any fully specified $\operatorname{sgn}\{\beta, \gamma\}$ is potentially qualitatively falsifiable. This can be seen by considering the following. As before, let $\gamma=\mathrm{I}$ and $\pi=\beta^{-1}$.

The qualitative inverse. Given sgn $\beta, \operatorname{sgn} \pi$ is a qualitative inverse of $\operatorname{sgn} \beta$ if and only if there exist magnitudes for the entries of $\beta$, consistent with $\operatorname{sgn} \beta$, such that $\operatorname{sgn} \beta^{-1}=\operatorname{sgn} \pi$.

If $\pi=\beta^{-1}$, then it is required that $\beta \pi=\mathrm{I}$ and $\pi \beta=\mathrm{I}$. Accordingly, if $\operatorname{sgn} \pi$ is a qualitative inverse of $\operatorname{sgn} \beta$, then there must be magnitudes for the entries of $\beta$ and $\pi$ that lead to the identity matrix outcomes. It is "easy" to always formulate sign patterns for $\operatorname{sgn} \pi$, given $\operatorname{sgn} \beta$, such that $\beta \pi=I$ and/or $\pi \beta=$ I are impossible, independent of magnitudes. For example, if the entries of the first column of $\operatorname{sgn} \pi$ incident upon the non-zeros of the first row of sgn $\beta$ are in each and every case the negative of the corresponding entry of the first row of $\operatorname{sgn} \beta$, then it must be that

\footnotetext{
${ }^{13}$ In Keynesian style models the reduced form can be estimated by OLS, which is BLUE. If, as in modern macroeconomics, of which Sims (1986) is an early example, then the reduced form has additional properties such as super consistency.
} 


$$
\sum_{k=1}^{n} \beta_{1 k} \pi_{k 1}<0
$$

independent of magnitudes. ${ }^{14}$ Accordingly, the proposed sgn $\pi$ cannot be a qualitative inverse of the given sgn $\beta$. Indeed, any other proposed sgn $\pi$ with the signs of the entries of its first column so configured cannot be a qualitative inverse of the given sgn $\beta$, regardless of the signs of any other entries in the first column of sgn $\pi$, i.e., those corresponding to the zeros in the first row of $\beta$, or elsewhere in the array. Many other similar necessary requirements for row-column and column-row pairs of a given sgn $\beta$ and proposed sgn $\pi$ as required for the $\beta \pi=\mathrm{I}$ and $\pi \beta=\mathrm{I}$ impose similar limits on the sgn $\pi$ that can be the qualitative inverse of a given sgn $\beta$. For $\gamma \neq \mathrm{I}$, such restrictions remain on the possible $\operatorname{sgn} \beta^{-1}$ and lead to restrictions that are inherited by the possible sgn $\pi$. Even sign patterns for $\pi$ that satisfy these necessary conditions can nevertheless have other problems which make the proposed sgn $\pi$ impossible, independent of magnitudes. For example, impossible systems of inequalities for the entries of sgn adjoint $\beta$ may rule out a proposed sgn $\pi$. It is beyond our scope to further discuss how all such limits might be found. Instead, we note that such limits always exist. As a result, an estimated reduced form always has the potential to falsify the sign pattern hypothesized for the structural model.

IV. The Information Provided by the Hypothesis $\operatorname{sgn}\{\boldsymbol{\beta}, \gamma\}$. The definition of qualitative falsification only relates to the possible members of $\mathrm{RF}(\operatorname{sgn} \pi)$, which provide the information about the possible outcomes for the estimated sgn $\pi$ as related to potentially falsifying the hypothesis $\operatorname{sgn}\{\beta, \gamma\}$. The information provided by the hypothesis $\operatorname{sgn}\{\beta, \gamma\}$ can be measured using the Shannon entropy of the frequency distribution found for the members of RF(sgn $\pi)$. Let $F_{i}$ be the frequency of the $i^{\text {th }}$ sign pattern that appears in $R F(\operatorname{sgn} \pi)$ and $Q$ be the corresponding set of all such indices (how "i" can be assigned to a sign pattern is shown in the next section). Then,

$$
\operatorname{Entropy}(C Q(\beta, \gamma))=-\sum_{i \in Q} F_{i} \log \left(F_{i}\right)
$$

\footnotetext{
14 This is spelled out in Buck and Lady (2010).
} 
where $\log \left(\mathrm{F}_{\mathrm{i}}\right)$ is to the base 2. For our first example above, with only two possible sign patterns, each with a $50 \%$ chance of occurring, the corresponding measure of entropy is " 1 ." This measure is to be understood as follows: In general, an $\mathrm{n} \times \mathrm{m}$ pattern of signs, barring zeros, has $\mathrm{n} \mathrm{x} \mathrm{m} \mathrm{bits}$ of information. A bit for each entry, with (say) a value of " 0 " for a negative entry and a value of "1" for a positive entry. The "message" eventually received is the outcome of estimating $\pi$ and revealing its sign pattern. The entropy of the frequency distribution can be used to measure the information content of the "message," i.e., the amount of information that the frequency distribution does not provide that will be "learned" from the estimation of $\pi$. For example, if all possible $n$ by $m$ sign patterns were members of $\mathrm{RF}(\operatorname{sgn} \pi)$ and each was equally likely, then the entropy of the estimated sgn $\pi$ would be $n$ times $m$ (the maximum entropy). That is, a priori, the frequency distribution $\mathrm{F}_{\mathrm{i}}$ associated with $\operatorname{sgn}\{\beta, \gamma\}$ told us nothing about what to expect from the estimated sgn $\pi$. Alternatively, if only one sign pattern had been possible (with frequency $=1$ ), then the entropy of the estimated $\operatorname{sgn} \pi$ is zero; i.e., we already know the answer before receiving the "message." For our $2 \times 2$ example, the information provided by estimating $\pi$ contains only one bit (as determined using (5) above), i.e., the estimation shows whether it is the all negative or all positive case. The remaining information, i.e., that all entries of $\pi$ have the same sign, is already provided by the constraints on the members of $\mathrm{RF}(\operatorname{sgn} \pi)$ imposed by the hypothesis expressed by the sign patterns of $\{\beta, \gamma\}$.

The measure of entropy can be manipulated to reflect the information content of the hypothesis represented by the model, rather than what is left to be determined by the estimation of $\pi$. Accordingly, the information provided by the model is given by:

$$
\operatorname{INFO} \%(C Q(\beta, \gamma))=100\left(1-\frac{\operatorname{Entropy}(C Q(\beta, \gamma))}{n m}\right) .
$$

In our current example the hypothesis provides $75 \%$ of the four bits of information required to express the $2 \times 2$ sign pattern of $\pi=\beta^{-1}$. For this example, the hypothesis is falsified if any of the other fourteen conceivable sign patterns for $\pi=\beta^{-1}$ is exhibited by the data when $\pi$ is estimated. 
For the example where,

$$
\operatorname{sgn} \beta=\left[\begin{array}{ll}
- & ? \\
+ & -
\end{array}\right],
$$

the entropy of the corresponding frequency distribution using (5) is " 1.5 " and the information content of the posited $\mathrm{CQ}(\beta, \gamma)$ using $(6)$ is,

$$
\mathrm{INFO} \%(\mathrm{CQ}(\beta, \gamma))=62.5
$$

The entropy measure contains a fractional bit in the sense of an average, e.g., the average attendance last month to Tuesday's 11 AM lecture was 30.5 students.

It is "easy" to construct additional examples for larger systems, assuming for computational reasons, that they are kept sufficiently sparse in terms of the number of nonzero entries. For example, take the case of an irreducible matrix, $\beta$ (with $\gamma=\mathrm{I}$ ) with a negative main-diagonal and a single inference cycle involving all of the endogenous variables, e.g., with the only nonzero off-diagonal entries being those of the first lower sub-diagonal and $\beta_{1 n}$. This matrical form will have only two terms in the expansion of its determinant, the product of the main-diagonal entries and the product of the off-diagonal entries. As before, assume that the absolute values of the nonzero terms are randomly chosen from the uniform distribution, $0<\operatorname{abs}\left(\beta_{\mathrm{ij}}\right)<10$. For any value of $\mathrm{n}$ the adjoint of this matrical form is fully signed. Further, if the sign of the product of the off-diagonal entries is negative, then the sign of the determinant is always $(-1)^{\mathrm{n}}$. If the product of these entries is positive, then, when $\beta$ is nonsingular, the determinant is positive or negative, each half the time. For any value of $n$, the corresponding $\operatorname{RF}(\operatorname{sgn} \pi)$ has only two members, each appearing half the time for the value of the off-diagonal cycle positive. When the off-diagonal cycle is negative, there is just one member of $\mathrm{RF}(\operatorname{sgn} \pi)$.

The above examples notwithstanding, the algorithmic principles that enable any specification of $\{\beta, \gamma\}$ to be worked through to a specification of the members of $\operatorname{RF}(\operatorname{sgn} \pi)$ and their 
frequencies of occurrence can be problematic. For example, suppose $\mathrm{n}=\mathrm{m}=3, \gamma=\mathrm{I}, 0<\mathrm{abs}\left(\beta_{\mathrm{ij}}\right)$ $<10$, and,

$$
\operatorname{sgn} \beta=\left[\begin{array}{lll}
- & + & + \\
+ & - & + \\
+ & + & -
\end{array}\right] .
$$

Of the 512 conceivable sign patterns for $\beta^{-1}$, barring zeros, only nine of them are possible for the inverse of $\beta$ (when non-singular) with the above sign pattern. ${ }^{15}$ Looked at individually, each main diagonal cofactor, when non-zero, should be positive or negative half of the time. But, taken together, the signs of the main-diagonal cofactors are inter-correlated, since they share some entries of $\beta$ in common. Deriving the probability distribution of the members of $\operatorname{RF}(\operatorname{sgn} \pi)$ for this example or, for that matter, the members and frequencies of $\mathrm{RF}(\operatorname{sgn} \pi)$ for any system is case specific and for sufficiently large systems problematic. We did not attempt to formulate a method to solve this probability distribution problem. Instead, the Monte Carlo approach described in the next section was implemented. Using this simulation, based upon two million draws from $\operatorname{CQ}(\beta, \gamma)$, we found for the above $3 \times 3$ array that Entropy $(\operatorname{CQ}(\beta, \gamma))=3.1$ and $\mathrm{INFO} \%(\mathrm{CQ}(\beta, \gamma))=65.5$.

V. A Monte Carlo Approach for Investigating the Characteristics of RF(sgn $\pi)$. The Monte Carlo approach described in this section was used, for given $\operatorname{sgn}(\beta, \gamma)$, to provide estimates of the members of, and frequencies of occurrence of the members of, the corresponding $R F(\operatorname{sgn} \pi)$. The method is implemented by drawing many samples from $\operatorname{CQ}(\beta, \gamma)$, computing the corresponding $\pi$, and keeping track of the sign patterns found for $\pi$ and their frequencies. For the purpose of facilitating the development of the algorithm, we invoked assumptions that are not required for the proposed analytic point of view. Specifically, we assumed that $\beta$ was irreducible and that $\beta^{-1}$ and $\pi$ did not otherwise have zero entries. The reason for this was a simple practicality. We wanted to base the index system for sign patterns, as described below, on binary

\footnotetext{
${ }^{15}$ For this sign pattern all of the off-diagonal cofactors are positive. When non-zero, all of the on-diagonal cofactors can be positive or negative, each half of the time. If one on-diagonal cofactor is negative, then the determinant is positive. If all on-diagonal cofactors are positive, then, when non-singular, the determinant can be positive or negative; but, most of the time positive, since five of the six terms in the expansion of the determinant are positive.
} 
numbers, i.e., " 0 " for "_" and " 1 " for "+." Were zeros allowed, the index system would have to be based on base three numbers. There is nothing wrong with this; however, the binary numbers are easier to work with and there are many applied systems that conform to these additional assumptions. ${ }^{16}$

Our method is as follows:

(i) The sign patterns of $\{\beta, \gamma\}$ are specified, including nonzeros with uncertain signs.

(ii) For a single trial, $\operatorname{CQ}(\beta, \gamma)$ is sampled as the values of the nonzero entries chosen in the range $0<\left|\beta_{\mathrm{ij}}, \gamma_{\mathrm{ij}}\right|<10$. The sign pattern of the nonzero entries is then applied. Nonzeros with unknown signs are set positive or negative each half of the time. ${ }^{17}$

(iii) If there is additional information about the entries in the two arrays, this is now imposed, e.g., the entries in accounting equations are often " 1 " or "-1." As discussed below, for purposes of falsification, there are advantages to skipping this step.

(iv) Given the quantitative realizations of $\{\beta, \gamma\}, \pi=\beta^{-1} \gamma$ is computed.

(v) Given this result, the resulting sign patterns of $\pi$ 's individual entries, rows, columns, and of $\pi$ itself are recorded. For each row or column of a particular $\pi$, the sign pattern found is expressed as a binary number with " 0 " for negative and " 1 " for positive signs. The base 10 number corresponding to this binary number is computed and used as the index for the sign pattern found. For $\pi$ in its entirety, the binary number used is formed by writing out the sign pattern of all of its rows written end-to-end as a row vector. The base 10 number corresponding to this binary number is the index of the sign pattern found for the entirety of $\pi$.

\footnotetext{
${ }^{16}$ In any case, using base two does not limit the analysis in its general application. We present our method because we used it to work with the examples given below. For smaller systems, its convenience is obvious. Still, the paper and the analytic point of view presented is not about the choice of logarithmic base nor do we necessarily advocate it compared to other methods that might be developed.

${ }^{17}$ A similar method was reported on in Lady and Sobel (2006). In that application only a tabulation of signs of the entries of $\beta^{-1}$ was recorded.
} 
(vi) For each sign pattern index observed, increment the corresponding frequency.

(vii) Stop if the preset number of samples, V, has been reached, or otherwise return to (i).

To summarize the algorithm, for a single simulation, the number of samples $\mathrm{V}$ is usually set in the hundreds of thousands, or even millions. As the simulation is under way, the sign patterns for each row and column of $\pi$ that appeared and their frequency of occurrence are tabulated. For sufficiently small systems ${ }^{18}$ (say, $\mathrm{nx} \mathrm{m}<26$ ), the sign patterns of $\pi$ itself and their frequency of occurrence are also tabulated and the information and entropy measurements outlined above are computed.

For purposes of falsification, the sign pattern of a particular data-based estimate of $\pi$, denoted $\hat{\pi}$, is observed. If the signs of any of $\hat{\pi}^{\prime}$ s rows or columns, or of $\hat{\pi}$ itself, do not appear across sometimes millions of samples of $\operatorname{CQ}(\beta, \gamma)$, then the proposed model is, or at least appears to be, falsified. This, even though no individual sign of the reduced form failed to appear. Paradoxically, a disallowed $\hat{\pi}$ might even be solved for the corresponding $\hat{\beta}$ and $\hat{\gamma}$ yielding signs and statistical significance that are plausible from the standpoint of the original hypothesis about the structural model. For a $\hat{\pi}$ that is not found by the Monte Carlo, the symbolic expansion of terms can be examined to confirm that the sign pattern found from the data is not actually possible given the hypothesized $\beta$ and $\gamma$.

Returning to the design of the Monte Carlo, specific quantitative values on any of the entries as identified in step (iii) above need not be imposed even when these are known. When only processing the sign patterns of the arrays, then the simulation can draw any member of $\operatorname{CQ}(\beta, \gamma)$ subject to scaling. For example, take any $\beta$ whatsoever. Let MAX be the largest absolute value of any of its entries. Given this, form $\beta^{*}$ by multiplying each entry of $\beta$ by, say, (1/MAX). The sign pattern of the inverse of $\beta^{*}$ is the same as that of $\beta$. And, even if $\beta$ cannot be sampled due to the distributional rules specified for the values of the entries of $\beta, \beta^{*}$ can be. As a result, the

\footnotetext{
${ }^{18}$ In principle the system can be of any size. We were limited by the size and speed of our computer.
} 
member of $\mathrm{RF}(\operatorname{sgn} \pi)$ corresponding to $\beta$ will not (necessarily) be missed by our sampling procedure. Specifically, the members of $\operatorname{RF}(\operatorname{sgn} \pi)$ with restrictions added in (iii) above will also be members of $\mathrm{RF}(\operatorname{sgn} \pi)$ without the restrictions added. Accordingly, if a row, column, or entire sign pattern of $\pi$ does not appear without the restrictions in (iii) added, it would not appear with the restrictions added. The same conclusion holds for the posited sign pattern of $\gamma$, its rescaling, and the possible outcomes for the sign pattern of $\pi$.

The "risk" of the method is to fail to find a member of RF(sgn $\pi)$ that actually exists, but only with an extremely small frequency. Failing to find a member of $\mathrm{RF}(\operatorname{sgn} \pi)$ could result in incorrectly estimating the entropy of the system and misjudging the information content of the proposed model. Also, failing to find a member of $\mathrm{RF}(\operatorname{sgn} \pi)$ could result in incorrectly falsifying a model, which would be tantamount to a Type I error in classical statistics. There are three parts to the response to this issue. First, since the Monte Carlo method used is essentially a process by which the empirical probability distribution of sign patterns is built up, one must explore the statistical properties of this distribution estimator. Second, the estimator for the entropy of the model should have desirable statistical properties as well. Third, even if the empirical density has desirable statistical properties, what is known about the probability of the Monte Carlo method missing a possible outcome of RF( $\operatorname{sgn} \pi)$ ?

The possible sign patterns for a given row or column of $\pi$, or even the entire matrix, is a multinomial distribution with unknown proportions. The Monte Carlo method used to generate the data on the proportions of sign patterns of $\pi$ is a maximum likelihood estimator. As a class, maximum likelihood estimators are known to be unbiased estimators for the first moment, the case here. They are also known to be efficient and consistent.

Regarding the second point, from equation (5) $\operatorname{Entropy}(C Q(\beta, \gamma))=-\sum_{i \in Q} F_{i} \log \left(F_{i}\right)$ is an estimate of the entropy of a system calculated from a sample of size $\mathrm{V}$ (the number of repetitions in the Monte Carlo), an event set of q outcomes of $\pi$ determined by the number of indices in the set $\mathrm{Q}$ (i.e., the number $\mathrm{F}_{\mathrm{i}}$ that are not zero) and $\mathrm{F}_{\mathrm{i}}$ is the observed relative frequency of a member of the event set. The mean and variance of the entropy estimator (Basharin(1959)) are 


$$
\begin{gathered}
\mathrm{E}(\hat{\operatorname{Entropy}}(\bullet))=\operatorname{Entropy}-\frac{\mathrm{q}-1}{\mathrm{~V}} \log (\mathrm{e})+\mathrm{O}\left(1 / \mathrm{V}^{2}\right) \\
\operatorname{Var}(\hat{\operatorname{Entropy}}(\bullet))=\frac{1}{\mathrm{~V}}\left(\sum_{\mathrm{i} \in \mathrm{Q}} \mathrm{F}_{\mathrm{i}}\left(\log \left(\mathrm{F}_{\mathrm{i}}\right)\right)^{2}-\operatorname{Entropy}(\bullet)^{2}\right)+\mathrm{O}\left(\frac{1}{\mathrm{~V}^{2}}\right)
\end{gathered}
$$

The entropy estimator underestimates the actual entropy, but the bias always can be made smaller by choosing the sample size to be larger, and it vanishes in the limit. ${ }^{19}$ Also, since the sample size is in the denominator of the variance, the entropy estimator is statistically consistent.

The final issue is the probability of missing the $\mathrm{i}^{\text {th }}$ sign pattern in the Monte Carlo experiment. Chebyshev's inequality, $\operatorname{Pr}\left(\left|F_{i}-E F_{i}\right|>t\right) \leq \frac{\sigma_{i}^{2}}{t^{2}}$, can be used to put an upper bound on that probability. " $\mathrm{t}$ " is an arbitrarily chosen small number and $\sigma_{\mathrm{i}}^{2}$ is the variance of the $\mathrm{i}^{\text {th }}$ parameter in the multinomial process that generates the data. The variance for the $i^{\text {th }}$ term is given by $\frac{F_{i}\left(1-F_{i}\right)}{V}$. Since the number of samples is in the denominator, the probability of missing a sign pattern can be made smaller by making the number of repetitions larger. ${ }^{20}$.

\section{Examples}

Klein's Model I. Klein's model I (Klein (1950)) is an over-identified ${ }^{21}$ econometric $^{-1}$ model of the U.S. economy. It has been considered in the literature for a variety of

\footnotetext{
${ }^{19}$ Or at least it gets sufficiently small in principle. For larger systems, the required number of samples may be large compared to the available computing capability.

${ }^{20}$ For large systems, notwithstanding these observations, the magnitudes of the number of possible sign patterns for $\pi$ (even barring zeros), the possible degree to which some sign patterns might be unlikely, and a correspondingly sensible size for the number of samples taken are all issues that would benefit from future innovation.

${ }^{21}$ A model is over-identified if the estimable reduced form provides too many linearly independent equations in the unknowns of the structural model.
} 
methodological and pedagogical purposes. ${ }^{22}$ Maybee and Weiner (1988), and later Lady (2000), analyzed the qualitative properties of $\beta$ (for the model expressed in the form of (3) above). Both of these efforts were intended to demonstrate how to cope with the fact that the sign pattern for $\beta$ as proposed for the model did not submit to a successful qualitative analysis. Instead, it was shown how to use additional, quantitative information in signing some of the entries of $\beta^{-1}$. None of this was focused on testing if the model's specification survived an estimation of the reduced form as was done in Buck and Lady (2005).

Absent an error vector, Klein's model is given by,

$$
\begin{gathered}
\beta Y=\gamma Z, \text { where } \\
{\left[\begin{array}{ccccccc}
-1 & 0 & a_{1} & 0 & a_{2} & 0 & 0 \\
0 & -1 & 0 & 0 & b_{1} & 0 & 0 \\
0 & 0 & -1 & 0 & 0 & 0 & c_{1} \\
1 & 1 & 0 & -1 & 0 & 0 & 0 \\
0 & 0 & 0 & 1 & -1 & -1 & 0 \\
0 & 0 & 1 & 0 & 0 & -1 & 0 \\
0 & 0 & 0 & 1 & 0 & 0 & -1
\end{array}\right]\left[\begin{array}{c}
C \\
I \\
W_{1} \\
Y \\
P \\
W \\
E
\end{array}\right]=\left[\begin{array}{ccccccc}
-a_{1} & -a_{3} & 0 & 0 & 0 & 0 & 0 \\
0 & -b_{2} & -b_{3} & 0 & 0 & 0 & 0 \\
0 & 0 & 0 & -c_{2} & -c_{3} & 0 & 0 \\
0 & 0 & 0 & 0 & 0 & 1 & -1 \\
0 & 0 & 0 & 0 & 0 & 0 & 0 \\
-1 & 0 & 0 & 0 & 0 & 0 & 0 \\
1 & 0 & 0 & 0 & 0 & -1 & 0
\end{array}\right]\left[\begin{array}{c}
W_{2} \\
P_{-1} \\
K_{-1} \\
E_{-1} \\
Y e a r \\
T X \\
G
\end{array}\right]}
\end{gathered}
$$

In Klein's model the endogenous variables are private consumption (C), investment (I), the private wage bill $\left(\mathrm{W}_{1}\right)$, income $(\mathrm{Y})$, profits or nonwage income $(\mathrm{P})$, the sum of private and government wages (W), and private product (E); and the exogenous variables are the government wage bill $\left(\mathrm{W}_{2}\right)$, lagged profits $\left(\mathrm{P}_{-1}\right)$, end of last period capital stock $\left(\mathrm{K}_{-1}\right)$, lagged private product (E-1), years since 1931 (Year), taxes (TX), and government consumption $(\mathrm{G})$.

The sign patterns of the arrays proposed by Klein are as follows,

\footnotetext{
${ }^{22}$ Klein's model was the basis for much of the macroeconomic policy modeling spawned by the Cowles Foundation. Goldberger (1964), Berndt (1991) and Greene (2000) all used it pedagogically to demonstrate alternative econometric approaches for dealing with the identification problem at the time of estimation.
} 


$$
\operatorname{sgn} \beta=\left[\begin{array}{ccccccc}
- & 0 & + & 0 & + & 0 & 0 \\
0 & - & 0 & 0 & + & 0 & 0 \\
0 & 0 & - & 0 & 0 & 0 & + \\
+ & + & 0 & - & 0 & 0 & 0 \\
0 & 0 & 0 & + & - & - & 0 \\
0 & 0 & + & 0 & 0 & - & 0 \\
0 & 0 & 0 & + & 0 & 0 & -
\end{array}\right] \text { and sgn } \gamma=\left[\begin{array}{ccccccc}
- & - & 0 & 0 & 0 & 0 & 0 \\
0 & - & + & 0 & 0 & 0 & 0 \\
0 & 0 & 0 & - & - & 0 & 0 \\
0 & 0 & 0 & 0 & 0 & + & - \\
0 & 0 & 0 & 0 & 0 & 0 & 0 \\
- & 0 & 0 & 0 & 0 & 0 & 0 \\
+ & 0 & 0 & 0 & 0 & - & 0
\end{array}\right] \cdot{ }^{23}
$$

The estimated coefficient (sign pattern) of the reduced form of Klein's model is reported in Goldberger (1964) as

$$
\text { Estimated } \operatorname{sgn} \hat{\pi}=\left[\begin{array}{lllllll}
- & + & - & + & + & - & + \\
- & + & - & - & + & - & + \\
- & + & - & + & + & - & + \\
- & + & - & + & + & - & + \\
- & + & - & + & + & - & + \\
- & + & - & + & + & - & + \\
- & + & - & + & + & - & +
\end{array}\right] \text {. }
$$

The issue of falsification of Klein's original specification using its reduced form can be visited in terms of the limitations on the members of $\mathrm{RF}(\operatorname{sgn} \pi)$ imposed by the structural hypothesis presented by the sign patterns of $\{\beta, \gamma\}$ in (7). The sign pattern of the entire reduced form itself is expressed by 49 bits. This made the range of base 10 indices for the sign patterns that could be taken on by the entire reduced form matrix $\pi$ to be from 0 to $\left(2^{49}-1\right)$. The upper values in this range were too large to represent as integers within the processing platform we were using for the Monte Carlo simulation. As a result, we did not tabulate the occurrence of entire sign patterns of the reduced form for each $\{\beta, \gamma\}$ drawn. Instead, we tabulated the sign patterns for each row and each column of $\pi$. Each of these is expressed by seven bits; so that there are 128 possible sign patterns for each row and each column of $\pi .^{24}$

\footnotetext{
${ }^{23}$ All of the unknown entries were hypothesized to be positive except $b_{3}$, which was hypothesized to be negative. As a result, the hypothesis is $\gamma_{23}>0$.

${ }^{24}$ We did conduct a "simple search" by sampling $\{\beta, \gamma\}$, computing $\pi=\beta^{-1} \gamma$, and then checking to see if the sign pattern found conformed to that given in Goldberger, our equation (8), all without keeping track as to what sign
} 
Inspection of the specification of the model above shows that there are only 27 non-zeros in the arrays $\{\beta, \gamma\}$. Of these, ten are estimated. The remaining are either " 1 " or "-1" appropriate to accounting relationships among the model's endogenous and exogenous variables. We wanted to take this information into account; however, we were concerned about the scaling issue noted above, since we had no basis for setting the bounds on the other estimated entries, given that some entries were pegged to be " 1 " or "-1." To avoid this issue, the absolute value of $\beta_{11}$ (which by definition is equal to -1 ) was chosen in the open interval $-10<\beta_{11}<0$ and then, all of the other entries equal to " 1 " or "- 1 " per the above specification, were set equal to $\beta_{11}$ with the appropriate signs applied. Each time the simulation was run for $1,000,000$ draws from $\operatorname{CQ}(\beta, \gamma)$ subject to the above rules. ${ }^{25}$ Below are the results for one simulation for a sample of 1,000,000 draws for $\{\beta, \gamma\}$. The results are presented first for the row sign patterns found for the reduced form (Table 1), then for the column sign patterns found (Table 2); and finally, a comparison of these findings to the unrestricted reduced form estimation results, given in (8) above, is displayed in (Table 3).

patterns were otherwise found. The sign pattern given in Godberger was never found. As given below, we checked the algebra for $\pi=\beta^{-1} \gamma$ and found out why.

${ }^{25}$ Actually, many millions of trials were run while developing the simulator, all with the same results, as given in tables 1 and 2 below. 
Table 1.

Reduced Form Sign Pattern Frequency Distributions By Row for Sign Patterns That Appeared At Least Once

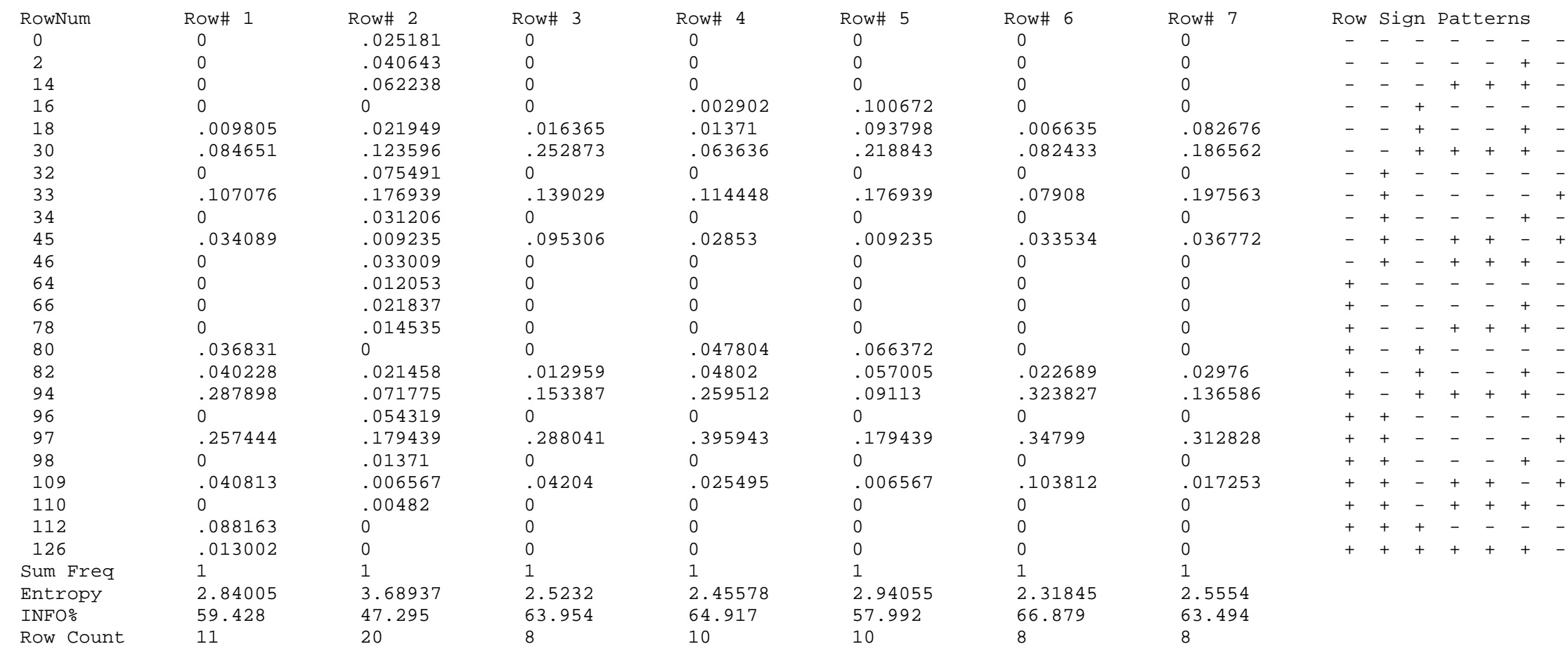


Table 2.

Reduced Form Sign Pattern Frequency Distributions By Column for Sign Patterns That Appeared At Least Once

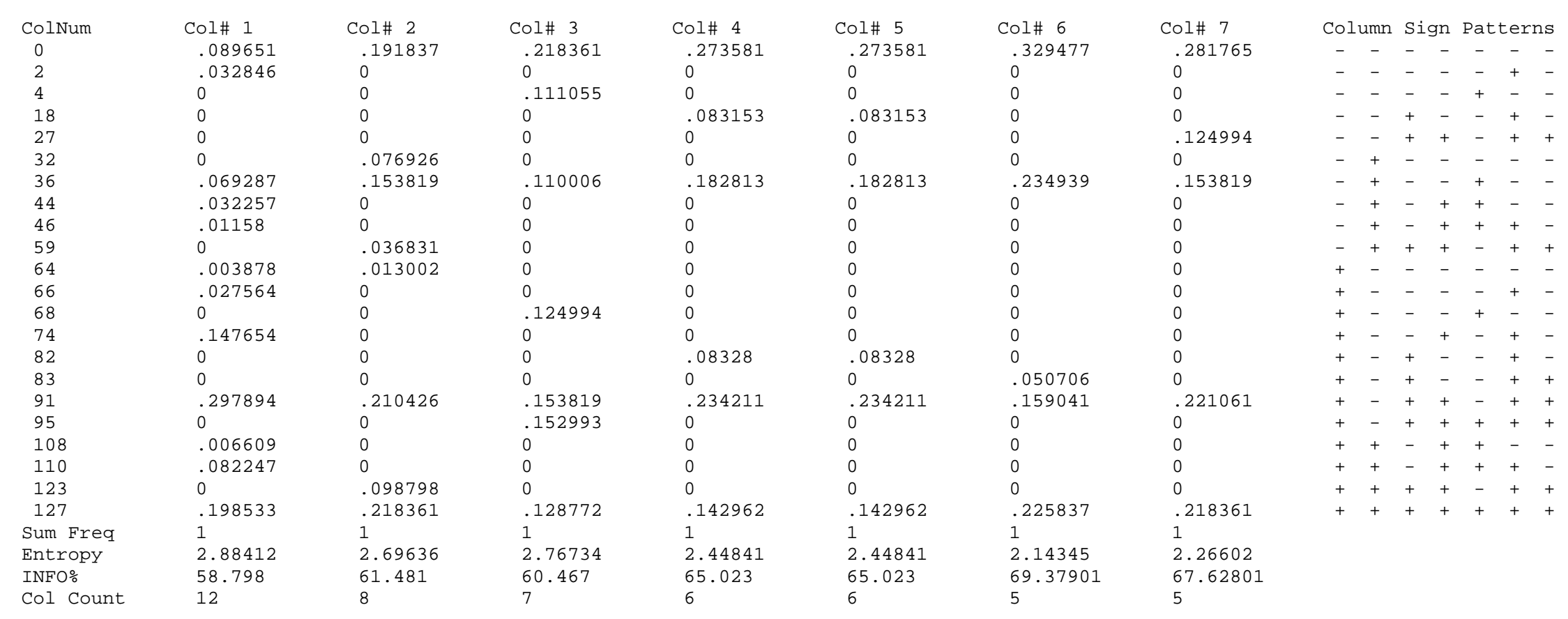


In tables 1 and 2, above, the first column gives the base 10 index of a row or column sign pattern that appeared at least once as a member of $\mathrm{RF}(\operatorname{sgn} \pi)$ corresponding to a sample of $1,000,000$ trials of the quantitative realizations of $\{\beta, \gamma\}$ as described above. The next seven columns show the frequency with which the given sign pattern appeared for each row (Table 1) or column (Table 2). The last display or panel of each table is the sign pattern itself. The last rows show the sum of the frequencies (an error check); the entropy of the frequency distributions for each row or column using (5) above; the corresponding information content of the structural form for each row or column using (6) above; and finally, the number of sign patterns that were found for each row and column out of the 128 possibilities for a pattern of seven signs, barring zeros.

Although the appearance of entire specific reduced form sign patterns was not cataloged due to the problem of assigning a base 10 index to all of the possible 7 by 7 sign patterns, barring zeros, the cataloging of sign patterns for rows and columns allows an upper bound to be placed on the total number of members of RF(sgn $\pi)$. For example, assuming that the sign patterns found for each column as given in Table 2 are the only possible sign patterns, given the hypothesis (3) for Klein's model, then at most the number of possible reduced forms would be the product of the numbers found for each column: $12 \times 8 \times 7 \times 6 \times 6 \times 5 \times 5=604,800$. This outcome follows if the sign pattern for each column appears independent of the sign patterns for other columns (which it almost surely does not). For this upper bound, entropy is maximized if each possibility is equally likely. The value of the entropy for this case is the log base 2 of the upper bound on the possible sign patterns for $\pi, \log _{2}(604800)=19.2$. Applying $(6)$ to this result, the lower bound on the amount of information provided by the hypothesis is INFO $\%=60.8$. As it works out for this example, this result using the data on columns found is the binding result, compared to that for rows, since fewer possible reduced forms are allowed by the numbers of column sign patterns found.

In Table 3, below, the sign patterns of the row and columns of the estimated reduced form found from the 1921-1941 annual data given in (8) are compared to the sign patterns found by the simulation. 
Table 3. Simulation Results for the Un-Restricted Klein Model 1 Reduced Form

\begin{tabular}{|c|c|c|c|c|c|c|c|c|}
\hline & W2 & P-1 & K-1 & E-1 & Year & TX & G & FREQ. \\
\hline C & - & + & - & + & + & - & + & .0341 \\
\hline I & - & + & - & - & + & - & + & 0 \\
\hline W1 & - & + & - & + & + & - & + & .0953 \\
\hline Y & - & + & - & + & + & - & + & .0285 \\
\hline P & - & + & - & + & + & - & + & .0092 \\
\hline W & - & + & - & + & + & - & + & .0335 \\
\hline E & - & + & - & + & + & - & + & .0368 \\
\hline FREQ & .0897 & .2184 & .2184 & 0 & .1430 & .3295 & .2184 & \\
\hline
\end{tabular}

In the body of Table 3 the sign pattern of the unrestricted reduced form given in (8) above is reiterated. The entries in the last column give the frequencies with which the row sign pattern appeared in the sample of 1,000,000 trials reported on in Tables 1 and 2. The entries in the last row give the same information for each column. Notably, the sign pattern found for row two of the estimated reduced form was not found by the simulation nor was the sign pattern for column four. These results falsify the hypothesized sign patterns given above for the structural arrays $\{\beta$, $\gamma$ \} proposed by Klein.

For row two, inspection of the row sign patterns found by the simulation revealed that in no case $\operatorname{did} \pi_{24}$ and $\pi_{25}$ have opposite signs, as called for by the unrestricted reduced form estimate of (8). A quick check of the algebra for $\pi=\beta^{-1} \gamma$ revealed that:

$$
\pi_{24}=\left[\beta^{-1}\right]_{23} \gamma_{34} \text { and } \pi_{25}=\left[\beta^{-1}\right]_{23} \gamma_{35}
$$

Since $\gamma_{34}$ and $\gamma_{35}$ are both negative, $\pi_{24}$ and $\pi_{25}$ cannot have opposite signs, independent of magnitudes. This circumstance falsifies the hypothesis.

For column four, inspection of the column sign patterns found by the simulation revealed that in no case did $\pi_{24}$ and $\pi_{54}$ have opposite signs, as was found for the unrestricted reduced form estimate of (8). For these entries of $\pi$,

$$
\pi_{24}=\left[\beta^{-1}\right]_{23} \gamma_{34} \text { and } \pi_{54}=\left[\beta^{-1}\right]_{53} \gamma_{34}
$$


From the simulations of $\beta^{-1}$ there was evidence that $\left[\beta^{-1}\right]_{23}$ and $\left[\beta^{-1}\right]_{53}$ have the same sign, independent of magnitudes. The evidence was in the form of positive entries for those terms with equal and high frequency (0.79832). For many different Monte Carlo simulations, the frequency of positive values for these entries was always the same.

Since $\beta$ is a sparse matrix it was possible to write out its inverse and determine an analytic explanation for the falsification based on the non-occurrence of the observed estimated reduced form in the Monte Carlo simulations. ${ }^{26}$ The hypothesis specified that $b_{1}>0$, therefore it must be the case that $\left[\beta^{-1}\right]_{23}$ and $\left[\beta^{-1}\right]_{53}$ have the same sign since one is just a multiple of the other, and hence $\pi_{24}$ and $\pi_{54}$ also have the same sign. Inspection of the symbolic expansions of the terms in $\beta^{-1}$ shows many instances when certain sets of entries in $\beta^{-1}$ must have the same sign. Moreover, apart from the sign of the determinant (which is contingent on the magnitudes of the unknown a's, b's and c's) there are many instances of same sign restrictions in $\pi$ for which the Monte Carlo provided ample evidence and which restrictions were not imposed at the time of estimating the reduced form. In more complex systems this sort of post hoc analytic examination would be extremely difficult; still, the Monte Carlo method outlined here provides evidence that supports such an analysis.

An important question is that of how the proposed procedure for assessing a model's consistency with the data, as based on Monte Carlo simulation of model sign patterns outlined here, brings more to the analytic table than the classical econometric approach. Consider the pedagogy found in Goldberger(1964) and which is propagated and expanded upon in Berndt(1990) and Greene(2008).

In the empirical results reported by Goldberger(1964, pp. 325 and 368) the difference between the unconstrained reduced form estimates and the reduced form derived from the constrained ML estimates of the structural model differ in sign in eight out of forty nine instances, all involving only three of the seven exogenous variables. There are differences in magnitude (i.e. one coefficient estimate is two or more times as large as the other) for seventeen out of forty one

\footnotetext{
${ }^{26}$ These are available from the authors.
} 
cases where the unrestricted and constrained coefficients have the same sign, all involving only four of the seven exogenous variables. Noting "there are substantial differences in parameter estimates based on the unrestricted and restricted reduced form estimation," Berndt(1991) takes these differences as warranting a statistical test of the zero restrictions in the matrix $\gamma$ of the structural model. In a joint test of the restrictions Berndt rejects the hypothesis, but does not argue that the model has been falsified.

Reasoning from the multiplicity of estimates (contingent on the estimator used) of the structural parameters, Greene (2008) comes to the same conclusion that a test of the zero restrictions is necessary. He finds that the zero restrictions are rejected only for the third or wage equation of the model and subsequently argues that that equation may be mis-specified. Like Berndt, he does not argue that the model has been falsified.

Having found a problem with the zero restrictions, neither author notes that there is only one restricted-unrestricted reduced form pair that are statistically different from one another. This begs the question of whether the conduct of the test of hypothesis regarding the zero restrictions was motivated by their pedagogical interest or was a prescriptive test based on the observed empirical results. In any case, their approach leaves open the question of whether the model has been falsified. Comparatively, the results we present here are decisive: The sign pattern of the estimated reduced form is impossible, given the hypothesized sign patterns for $\{\beta, \gamma\}$.

Sims SVAR. By the end of the 1970s much was made of the poor forecasting performance of the many Keynesian macro-econometric models in use at that time. Sims (1980) nicely summarizes the state of the science and proposes an alternative vector autoregression approach. In the paper Sims roundly criticizes the over-identifying restrictions of traditional macro-econometric models, but states that the Klein type effort will linger on because of its usefulness for more structured forecasting and policy analysis. However, in Sims (1986) he makes a case for using structural vector autoregressions (SVAR) to model policy without the same level of a priori identifying restrictions used by Klein and his descendents. As a matter of the empirical implementation of economic theory Klein and Sims are quite distinct in their approaches. From the methodological perspective developed here the essential difference 
between Klein and Sims is that of how the identifying restrictions are imposed and their implication for admissible reduced forms. Klein and those following him invoked considerable $a$ priori economic reasoning regarding the exogenous variables in order to identify their models. Sims and those that have followed him have invoked less economic theory and imposed covariance restrictions on the structural error covariance matrix in order to achieve model identification. To reiterate the point made elsewhere in the paper, both Klein and Sims (and their legions of followers) can point to statistically significant results for their structural coefficients and neither has an approach to falsify the other's or their own model; and further, neither has presented a decisive case for their view of the world.

Sims (1986) offers two SVAR representations of how the economy works: Version 1 is

$$
\left[\begin{array}{cccccc}
1 & \beta_{11} & 0 & 0 & 0 & 0 \\
\beta_{21} & 1 & \beta_{23} & \beta_{24} & 0 & 0 \\
\beta_{31} & 0 & 1 & 0 & 0 & \beta_{36} \\
\beta_{41} & 0 & \beta_{43} & 1 & 0 & \beta_{46} \\
\beta_{51} & 0 & \beta_{53} & \beta_{54} & 1 & \beta_{56} \\
0 & 0 & 0 & 0 & 0 & 1
\end{array}\right]\left[\begin{array}{c}
r \\
m \\
y \\
p \\
u \\
i
\end{array}\right]=\Gamma Z+U
$$

And version 2 is

$$
\left[\begin{array}{cccccc}
1 & \beta_{11} & 0 & 0 & 0 & 0 \\
\beta_{21} & 1 & \beta_{23} & \beta_{24} & 0 & \beta_{26} \\
\beta_{31} & 0 & 1 & 0 & 0 & \beta_{36} \\
\beta_{41} & \beta_{42} & \beta_{43} & 1 & 0 & 0 \\
\beta_{51} & 0 & \beta_{53} & \beta_{54} & 1 & \beta_{56} \\
0 & 0 & 0 & 0 & 0 & 1
\end{array}\right]\left[\begin{array}{c}
r \\
m \\
y \\
p \\
u \\
i
\end{array}\right]=\Gamma Z+U
$$

In both models the $\beta_{\mathrm{ij}}$ are unknown coefficients to be estimated. The endogenous variables are the interest rate (r), money (m), output (y), prices (p), unemployment (u) and investment (i). In Sims' analysis the variables $\mathrm{m}, \mathrm{y}, \mathrm{p}$ and $\mathrm{i}$ are measured in logs, and $\mathrm{r}$ and $\mathrm{u}$ are in levels. On the right hand side are distributed lags over the endogenous variables, denoted by $Z$. The autoregressive coefficients of $\Gamma$ are of no particular importance and are not constrained by 
economic theory in either sign or magnitude. ${ }^{27} \mathrm{U}$ is a white noise error vector of dimension six with diagonal covariance matrix $\mathrm{E}\left(\mathrm{UU}^{\prime}\right)=\Sigma$. It is the zero contemporaneous covariances that allow Sims to identify either model with the use of the reduced form. The estimates for the $\beta_{\mathrm{ij}}$ are found as the solutions to the system

$$
\beta^{-1} \Sigma \beta^{\prime-1}=\hat{\Omega}
$$

where $\hat{\Omega}$ is the estimated reduced form error covariance matrix and $\Sigma$ is assumed to be the identity matrix. The results for $\hat{\Omega}$ are presented in Table 4 . The first column is the estimated covariance matrix using the data as transformed by Sims for the period 1948Q1 through 1979Q3. ${ }^{28}$ The error covariance matrix in the second column is obtained from the SVAR estimated with the same data, but all in levels. For the error covariance matrix in the third column a new data set was assembled for the period 1947Q1 through 2009Q3 and transformed following Sims. ${ }^{29}$ The changes in covariance signs contingent on the data source and transformation are surprising, but from the standpoint of falsification we remained agnostic.

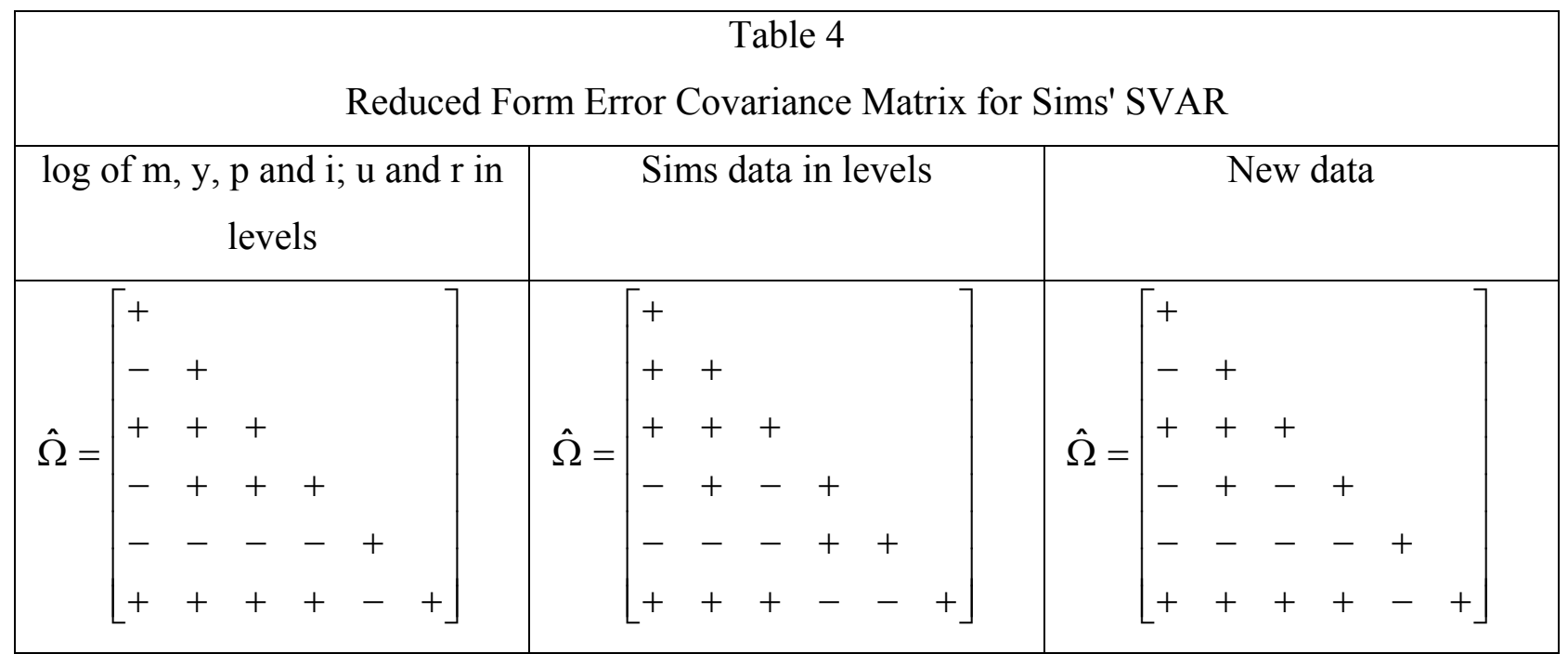

In the analysis of the Klein model the information content of the entire model could not be computed because of the problem of dimensionality. A lower bound on the information content

\footnotetext{
${ }^{27}$ The model must meet the stationarity constraints of any autoregressive process.

28 The Sims data was obtained from Awokuse and Bessler (2003).

${ }^{29}$ Using the larger data set all in levels did change the sign pattern of the error covariance matrix shown in column three.
} 
was reported as $60.8 \%$ in the earlier discussion. The outcome for Sims was constructed somewhat differently and was of considerable interest.

Following Sims (1986) we did not assume signs for the off-diagonal nonzeros in the above versions of the SVAR representations. We did set the main diagonal entries (assumed to be "1" in Sims' model) equal, but only at the simulated value for $\beta_{11}$, otherwise chosen randomly from the open interval $0<\operatorname{abs}\left(\beta_{11}\right)<10$ to accommodate the scaling problem noted above. The absolute values of the other nonzeros were also chosen from this interval, and then each set randomly as positive or negative, each half of the time. Since $\Omega$ is symmetric, although its sign pattern nominally contains thirty-six bits, its substantive sign pattern only contains twenty-one bits. Given this, barring zeros, there are 2,097,152 possible outcomes for the sign patterns of the estimated $\Omega$. This integer is "small" enough for our processing platform to keep track of all of the sign patterns found by the Monte Carlo for $\Omega$. As discussed, the form of $\beta$ assumed for the simulation only observed the zero restrictions of Sims' model, with the exception of setting the main diagonal entries positive and equal. We had wondered, a priori, as to the degree to which Sims' specification, as implemented this way, would limit the outcomes for the simulated $\Omega$, compared to the number possible. What we found dramatizes our basic thesis that fully specified sign patterns, and even in this case with hardly more than the zero restrictions applied, significantly limit the possible outcomes for their estimated realizations. For Sims, in tens of millions of trials implemented for both versions, the Monte Carlo consistently found only slightly more than $1 \%$ of the logically possible sign patterns. For the frequency distribution of outcomes actually found, this showed the information content of the model to be almost $56 \%$. If the outcomes found were assumed to be equally likely, the information content of the model still remained over $30 \%$ (the results for both versions were basically the same). The sign pattern for $\Omega$ found for each of the three data sets, as specified above in Table 4, was found by the simulation for each of the two specifications of Sims model. The results for Sims' Version 1, comparable to Klein, given in Table 5 below, report on the estimated sign pattern for $\mathrm{m}, \mathrm{y}, \mathrm{p}, \mathrm{I}$ as $\operatorname{logs}$ and $\mathrm{u}, \mathrm{r}$ as levels based upon a 2,500,000 iteration run of the Monte Carlo.

Table 5. Simulation Results for $\hat{\Omega}$, Sims Version 1 (m, y, p, I as $\operatorname{logs}$ and $u, r$ as level)

\begin{tabular}{|c|c|c|c|c|c|c|c|}
\hline & $\mathbf{r}$ & $\mathbf{m}$ & $\mathbf{y}$ & $\mathbf{p}$ & $\mathbf{u}$ & $\mathbf{i}$ & FREQ \\
\hline $\mathbf{r}$ & + & - & + & - & - & + & .0312 \\
\hline
\end{tabular}




\begin{tabular}{|c|c|c|c|c|c|c|c|}
\hline $\mathbf{m}$ & - & + & + & + & - & + & .0312 \\
\hline $\mathbf{y}$ & + & + & + & + & - & + & .0314 \\
\hline $\mathbf{p}$ & - & + & + & + & - & + & .0313 \\
\hline $\mathbf{u}$ & - & - & - & - & + & - & .0312 \\
\hline $\mathbf{i}$ & + & + & + & + & - & + & \\
\hline
\end{tabular}

This sign pattern was found only 152 times by the simulation. The sign pattern for the estimation using new data was found more often (805 times) and that based upon all level data was found less often (32 times).

As Sims himself proposes, compared to Klein, less is assumed about the direction of influence among the variables organized by the model. Nevertheless, the zero restrictions, and scarcely more, serve to substantially limit the possible outcomes for the estimated $\hat{\Omega}$; and, even so, Sims' model (both versions) is not falsified. How the information content of a model can be understood to trade-off with its consistency with the data is an interesting extension of our ideas, but remains beyond our scope in this paper.

The Oil Market Simulation (OMS). The OMS (System's Science, Inc. (1985), EIA (1990)) was used in the 1990's by the U.S. Department of Energy's Energy Information Administration (EIA) to make baseline forecasts of the world price of crude oil (WOP) in the preparation of their long term forecasts of U.S. energy production, distribution, and consumption. The model logic is straight-forward. World oil consumption is partitioned among some number of regions (seven in the version of the model used by EIA) with demand in each region represented by a log linear function (common in form to all demand regions) of the WOP and lagged regional demand. World, non-OPEC crude oil supply is also partitioned among some number of regions (eight in the version of the model used by EIA and not necessarily the same as those for demand) with supply in each region represented by a log linear function (common in form to all supply regions) of the WOP and lagged supply. Four more equations express the demand for OPEC production as the amount needed to balance the sum of regional supply and demand; plus, (nonlinear) relationships that express the WOP in terms of OPEC's rate of capacity utilization as related to maximum available capacity, as well as the lagged WOP. The general idea was that the WOP is larger as OPEC's current rate of capacity utilization is larger and the WOP was larger in the prior period. As used by EIA, the model had nineteen equations. 
An analysis of OMS' qualitative properties was conducted and reported on in Hale and Lady (1995) and reiterated in Hale, et al (1999). The purpose of the analysis was somewhat different than that used here. The effort was part of EIA's quality control program for its computer-based forecasting systems. To the degree to which an automated system had qualitative properties, then the direction of influence of changes in assumptions could be audited in terms of the consequent directions of change in the model's solution variables. As it worked out, the inverse Jacobian matrix of the OMS could be entirely signed; and accordingly, the qualitative results could be used to make sure that the computer implementation of the model was working properly.

Since this model is a rare instance of a model with qualitatively decidable entries in its reduced form (all of them in fact), and since it has been presented in the literature on qualitative analysis, we elected to use the model as an example here by assessing the model's consistency with the data. For our purposes we constructed a smaller version with only one demand region and only one (non-OPEC) supply region. The four equations relating to OPEC capacity utilization were retained, for six equations altogether.

Using our format, the differential analysis of the model's solution is expressed by,

$$
\left[\begin{array}{cccccc}
1 & 0 & 0 & 0 & 0 & \beta_{16} \\
0 & 1 & 0 & 0 & 0 & \beta_{26} \\
\beta_{31} & \beta_{32} & 1 & 0 & 0 & 0 \\
0 & 0 & \beta_{43} & 1 & 0 & 0 \\
0 & 0 & 0 & \beta_{54} & 1 & 0 \\
0 & 0 & 0 & 0 & \beta_{65} & 1
\end{array}\right]\left[\begin{array}{c}
d D \\
d S \\
d D O \\
d C A P U T \\
d R \\
d W O P
\end{array}\right]=\left[\begin{array}{cccc}
\gamma_{11} & 0 & 0 & 0 \\
0 & \gamma_{22} & 0 & 0 \\
0 & 0 & 0 & 0 \\
0 & 0 & \gamma_{43} & 0 \\
0 & 0 & 0 & 0 \\
0 & 0 & 0 & \gamma_{64}
\end{array}\right]\left[\begin{array}{c}
d D_{-1} \\
d S_{-1} \\
d M a x C a p \\
d W O P_{-1}
\end{array}\right],
$$

where D is world oil demand, S is non-OPEC world oil supply, DO is the demand for OPEC oil, CAPUT is the rate of OPEC capacity utilization, $\mathrm{R}$ is the percentage change (in decimal) of the current WOP over last year's, MaxCap is maximum OPEC capacity and WOP is the world oil price. The sign patterns for the arrays are given below, 


$$
\operatorname{sgn} \beta=\left[\begin{array}{cccccc}
+ & 0 & 0 & 0 & 0 & + \\
0 & + & 0 & 0 & 0 & - \\
- & + & + & 0 & 0 & 0 \\
0 & 0 & - & + & 0 & 0 \\
0 & 0 & 0 & - & + & 0 \\
0 & 0 & 0 & 0 & - & +
\end{array}\right] \text {, and } \operatorname{sgn} \gamma=\left[\begin{array}{cccc}
+ & 0 & 0 & 0 \\
0 & + & 0 & 0 \\
0 & 0 & 0 & 0 \\
0 & 0 & - & 0 \\
0 & 0 & 0 & 0 \\
0 & 0 & 0 & +
\end{array}\right] \text {. }
$$

For the given $\operatorname{sgn} \beta$, the sign pattern of $\beta^{-1}$ can be entirely determined and is given by,

$$
\operatorname{sgn} \beta^{-1}=\left[\begin{array}{cccccc}
+ & + & - & - & - & - \\
+ & + & + & + & + & + \\
+ & - & + & - & - & - \\
+ & - & + & + & - & - \\
+ & - & + & + & + & - \\
+ & - & + & + & + & +
\end{array}\right] \text {. }
$$

Further, the inverse Jacobian can be fully signed for any number of demand and supply regions so long as the associated demand and supply functions utilize the same log linear form. As a result, the reduced form for OMS can also be fully signed, as given below,

$$
\operatorname{sgn} \pi=\left[\begin{array}{cccc}
+ & + & + & - \\
+ & + & - & + \\
+ & - & + & - \\
+ & - & - & - \\
+ & - & - & - \\
+ & - & - & +
\end{array}\right] \text {. }
$$

This sign pattern is the only sign pattern that can be taken on by $\pi$, given the hypothesized sign patterns for $\beta$ and $\gamma$. Accordingly, the information content of the hypothesis is $100 \%$. If the hypothesis is true, nothing is to be learned by estimating the reduced form. Of course this 
circumstance makes the model vulnerable, in that all but one of the $2^{24}$-many possible $6 \times 4$ sign patterns (barring zeros) would falsify the model if estimated. ${ }^{30}$

Nevertheless, data were gathered from the EIA website for the years 1983-2006 and the reduced form of the differential form of OMS, as specified above, was estimated. The results are given below.

Table 6. OMS Estimated (1983-2006) Reduced Form Sign Pattern

\begin{tabular}{|c|c|c|c|c|}
\hline & $\mathrm{dD}_{-1}$ & $\mathrm{dS}_{-1}$ & $\mathrm{dMaxCap}$ & $\mathrm{dWOP}_{-1}$ \\
\hline $\mathrm{dD}$ & + & + & + & - \\
\hline $\mathrm{dS}$ & $-^{*}$ & + & - & $-^{*}$ \\
\hline $\mathrm{dDO}$ & + & $+^{*}$ & + & $+^{*}$ \\
\hline $\mathrm{dCaput}$ & $-^{*}$ & - & $+^{*}$ & $+^{*}$ \\
\hline $\mathrm{dR}$ & + & - & $+^{*}$ & - \\
\hline $\mathrm{dWOP}$ & + & - & - & $-^{*}$ \\
\hline
\end{tabular}

(“*”" indicates a "wrong" sign compared to the predicted reduced form sign pattern)

Inspection reveals that every equation in the reduced form has at least one "falsified" coefficient, except for total world demand. Altogether, nine entries of the reduced form, as estimated, are different in sign from their signs as allowed by the structural model.

Both OMS and Klein's Model I are specified and fully signed in the standard structural form. Accordingly, it is "easy" to re-specify the models to provide "less" information by both "unsigning" the coefficients to be estimated and implementing the SVAR methodology which does not account for the $\gamma$ array. This was done for both models. As it worked out, Klein's Model I was consistent with the data for the specifications involving less information. For OMS, given the qualitative invertibility of the $\beta$-matrix, the "weaker" specifications of OMS inherited a high information content from the signed, structural specification. Accordingly, all specifications of OMS were falsified. A summary of results is given in Table 7 below. In the table the reported "INFO\%" is measured for the frequency distribution of the arrays actually simulated by the Monte Carlo. The value reported for "Lower Bound INFO\%" assumes that the arrays found are

\footnotetext{
30 It should also be noted that the OMS model should be viewed as normative, rather than predictive. Oil is well traded in liquid spot and futures markets. Ex post, oil price time series would typically allow profitable arbitrage if known in advance. It really wouldn't make sense to predict such time series in advance (or admit it to others if you could). Accordingly, it should be no surprise that a baseline forecast constructed to support a forecasting model used for policy analysis fails to be predictive in particular, beyond base-line secular trends.
} 
equally likely. The information for Klein is based upon the column sign patterns found and the assumption of their independence, almost certainly an underestimate of the model's information content.

Table 7. Model Information Content and Consistency with the Data

\begin{tabular}{|c|c|c|c|}
\hline Model & INFO\% & $\begin{array}{c}\text { Lower Bound } \\
\text { INFO\% }\end{array}$ & $\begin{array}{c}\text { Consistency } \\
\text { With Data }\end{array}$ \\
\hline $\begin{array}{c}\text { KIein Stuctural } \\
\text { Signed }\end{array}$ & $\mathrm{n} / \mathrm{a}$ & 61 & Falsified \\
\hline $\begin{array}{c}\text { Klein Structural } \\
\text { Unsigned }\end{array}$ & $\mathrm{n} / \mathrm{a}$ & 37 & Consistent \\
\hline $\begin{array}{c}\text { Klein SVAR } \\
\text { Signed }\end{array}$ & $\mathrm{n} / \mathrm{a}$ & 42 & Consistent \\
\hline $\begin{array}{c}\text { Klein SVAR } \\
\text { Unsigned }\end{array}$ & $\mathrm{n} / \mathrm{a}$ & 37 & Consistent \\
\hline $\begin{array}{c}\text { Sims SVAR } \\
\text { Unsigned }\end{array}$ & 56 & 30 & Consistent \\
\hline $\begin{array}{c}\text { OMS Structural } \\
\text { Signed }\end{array}$ & 100 & 100 & Falsified \\
\hline $\begin{array}{c}\text { OMS Structural } \\
\text { Unsigned }\end{array}$ & 51 & 50 & Falsified \\
\hline $\begin{array}{c}\text { OMS SVAR } \\
\text { Signed }\end{array}$ & 73 & 67 & Falsified \\
\hline $\begin{array}{c}\text { OMS SVAR } \\
\text { Unsigned }\end{array}$ & 59 & 44 & Falsified \\
\hline
\end{tabular}

VII. Summary. This paper spells out how a model's structural specification can be used to assess the model's consistency with the data via its reduced form, i.e., determine if the limitations on the reduced form or its error covariance matrix implied by the structural model are actually observed. In addition the proposed method enables the model's information content to be measured, based upon Shannon's entropy concept, as it relates to the degree to which the model limits how the economy can perform. Examples are provided for a variety of model types and model specifications. For the included examples the more restrictive the structure, the more vulnerable is the model to falsification.

Some technical issues remain. These include the development of a means to work with entire arrays of larger dimension than was possible for our simulation. An important assumption in assessing a model's nature concerns the frequency distribution of values that might be taken on 
by its proposed structural parameters. This is in the nature of precise and diffuse priors in Bayesian statistics.

The approach outlined here enables qualitative principles about the interrelated directions of influence among economic variables, as well as even weaker specifications that do not specify sign patterns, to be applied to virtually any model. Altogether, there is considerable potential to very much increase an understanding of the information content of economic models; and, correspondingly, their scientific content and consistency, or not, with the data. 


\section{References}

Awokuse, Titus O. and David A. Bessler, 2003, "Vector Autoregressions, Policy Analysis, and Directed Acyclic Grapha: An Application to the U.S. Economy," Journal of Applied Economics, 6(1), Pps. 1-24.

Basharin, G.P. (1959), "On a statistical Estimate for the entropy of a sequence of independent random variables," Theory of Probability and its Applications, Vol 3, No 4, Pps. 333-336.

Bassett, Lowell, John Maybee, and James Quirk, 1968, "Qualitative Economics and the Scope of the Correspondence Principal," Econometrica, 36, pp. 544-563.

Berndt, Ernest, 1990, The Practice of Econometrics, Classic and Contemporary, New York, NY: Addison Wesley.

Buck, Andrew and George Lady, 2005, "Falsifying Economics Models," Economic Modelling, 22, September 2005, pp. 777-810.

, 2010, "An Expanded Scope for Qualitative Economics," DETU Working Paper 10_07, Department of Economics, Temple University.

Cover, Thomas and Joy Thomas, 1991, Elements of Information Theory, Wiley Series in Telecommunications, New York.

Energy Information Administration, 1990, “Oil Market Simulation User's Manuel, DOE/EIAM028(90), Washington, D.C.

Gerrard, Bill, 1995, "The Scientific Basis of Economics: A Review of the Methodological Debates in Economics and Econometrics," Scottish Journal of Political Economy, Vol. 42, No. 2, pp. 221-235.

Gilbert, C. 1986. 'Professor Hendry's Econometric Methodology'. Oxford Bulletin of Economics and Statistics 48: 283-307

Goldberger, Arthur S., 1964, Econometric Theory, New York: John Wiley and Sons.

Greene, William, 2008, Econometric Analysis, Upper Saddle River, New Jersey: Prentice Hall.

Hale, Douglas and George Lady, 1995, "Qualitative Comparative Statics and Audits of Model Performance" , Linear Algebra and Its Applications, 217, 1995, pp. 141-154.

Hale, Douglas, George Lady, John Maybee, and James Quirk, 1999, Nonparametric Comparative Statics and Stability, Princeton, New Jersey: Princeton University Press.

Hendry, David F., 1980, “Econometrics - Alchemy or Science?.” Economica, 47, pp. 387-406. 
Klein, Lawrence, 1950, Economic Fluctuations in the U.S., 1921-1941, Cowles Commission for Research in Economics Monograph No. 11, New York, NY, John Wiley and Sons.

Lady, George, 1983, “The Structure of Qualitatively Determinate Relationships,” Econometrica, 51, pp. 197-218.

, 1993, SGNSOLVE.EXE Analysis Package, prepared as a job of work for the Energy Information Administration, U.S. Department of Energy, Washington, D.C. , 2000, "Topics in Nonparametric Comparative Statics and Stability," International Advances In Economic Research, 5, pp. 67-83.

Lady, George and John Maybee, 1983, "Qualitatively Invertible Matrices," Mathematical Social Sciences, Vol. 6, 1983, pp. 397 - 407.

Lady, George and Marc Sobel, 2006, “Qualitative Inverses and Simulation,” Mimeo, Temple University.

Lady, George and James Quirk, 2007, "The Scope of the LeChatelier Principle," Physica A: Statistical Mechanics and its Applications, 381, pp. 351-365.

, 2010, "The Global LeChatelier Principle and Multimarket

Equilibria," The Review of Economic Design, 14, 1, pp. 193-201.

Lancaster, Kelvin, 1962, "The Scope of Qualitative Economics," Review of Economic Studies, 29, 99-132.

, 1966, "The Solution of Qualitative Comparative Statics Problems," Quarterly Journal of Economics, 53, pp. 278-295.

Leamer, Edward E., 1983, "Let's Take the Con Out of Econometrics," American Economic Review, 73(1), 31-43.

Maybee, John and Gerry Weiner, 1988, "From Qualitative Matrices to Quantitative Restrictions," Linear and Multilinear Algebra, 22, 229-248.

Maybee, Stuart, 1986, A Method for Identifying Sign Solvable Systems, M.S. theses, University of Colorado.

Metzler, Lloyd, 1945, "Stability of Multiple Markets: The Hicks Conditions," Econometrica, 13, 277-292.

Neffe, Jurgen (translated by Shelly Frisch), 2005 (Translation 2007), Einstein: A Biography, Farrar, Straus and Giroux, New York.

Pierce, John, 1980, An Introduction to Information Theory: Symbols, Signals, and Noise, $2^{\text {nd }}$ Edition, Dover Publications, Inc., New York. 
Popper, Karl , [1934] 1959, The Logic of Scientific Discovery, reprint, New York: Harper and Row.

Ritschard, Gilbert, 1983, "Computable Qualitative Comparative Statics Techniques," Econometrica, 51, 1145-1168.

Samuelson, Paul, 1947, Foundations of Economic Analysis, Cambridge: Harvard University Press. , 1960, “An Extension of the LeChatelier Principle," Econometrica, 28, 368-

379.

Shannon, C.E., 1948, "A Mathematical Theory of Communication," Bell System's Technical Journal, 27, 379-423, 623-656.

Sims, Christoper A., 1980, "Macroeconomics and Reality," Econometrica, 48(1), 1-48.

Sims, Christopher A., 1986, "Are Forecasting Models Usable for Policy Analysis," Federal Reserve Bank of Minneapolis Quarterly Review 10: 2-16.

System's Science, Inc., 1985, "The Oil Market Simulation Model, Documentation Report," DOE/EI/19656-2, Washington, D.C. 